\title{
The Application of Cumulants Slices in Fault Diagnosis
}

\author{
Wenbing Wu \\ Department of Mathmatics and Computer Science, Nanchang Normal University, Nanchang, China \\ Email: wwbysq@fjnu.edu.cn
}

How to cite this paper: Wu, W.B. (2018) The Application of Cumulants Slices in Fault Diagnosis. International Journal of Modern Nonlinear Theory and Application, 7, 89-96.

https://doi.org/10.4236/ijmnta.2018.73007

Received: July 7, 2018

Accepted: September 27, 2018

Published: September 30, 2018

Copyright $\odot 2018$ by author and Scientific Research Publishing Inc. This work is licensed under the Creative Commons Attribution International License (CC BY 4.0).

http://creativecommons.org/licenses/by/4.0/

(c) (i) Open Access

\begin{abstract}
Complex third-order cumulant has different definition forms. Different forms have different coupling properties, and the generated complex cumulants slices contain different coupling information of signals. In experiments, using the different definitions, the same coupling method is applied to both specific fault signals and normal signals. Furthermore, complex third-order cumulant slices spectrum is defined, and it is used to analyse the coupling features of normal signals and fault signals. Experiments indicate that the detection accuracy rate on the same fault is not the same with the different coupling method, thus, it provides an alternative method to diagnose the specific fault.
\end{abstract}

\section{Keywords}

Third-Order Cumulant Slices, Fault Diagnosis, Spectrum of Slices

\section{Introduction}

Using higher-order cumulant can suppress the gaussian background noise automatically (colored or white), because of this, the unify measurement of higher order cumulant has been brought to the people's attention and is becoming increasingly a very useful tool of signal processing. In general, the mechanical vibration signals are nonlinear, non-gaussian, the noise in the environment can be approximated as gaussian noise, and if vibration signals are analyzed by the high-order cumulant, it is easier to extract characteristic information related to the workpiece. Therefore, high order cumulant has been widely used in mechanical vibration, fault diagnosis and other fields [1] [2] [3] [4]. M.R. Raughveer and Wang Shuxun, domestic scholars, have conducted in-depth studies on the coupling properties of high-order cumulants, and clearly pointed out the coupling characteristics of various definitions forms of complex high-order 
quantities [5] [6] [7] [8]. In this article, based on this, fault diagnosis was made by using the complex third order cumulant slices. The experimental results show that, due to the fact that different definition in the form of the plural third-order cumulant slices contains the information of coupling, the different coupling information makes fault diagnosis more accurate, and illustrates that different coupling methods of plural third-order cumulant in keeping signals' features have different features.

\section{High Order Cumulant}

If $\{x(n)\}$ is a zero mean k-order stationary random process, then the k-order cumulative quantity $c_{k x}\left(\tau_{1}, \tau_{2}, \cdots, \tau_{k-1}\right)$ of this process is defined as the k-order cumulant of random variable $\left\{x(n), x\left(n+\tau_{1}\right), \cdots, x\left(n+\tau_{k-1}\right)\right\}$, namely:

$$
c_{k x}\left(\tau_{1}, \tau_{2}, \cdots, \tau_{k-1}\right)=\operatorname{cum}\left\{x(n), x\left(n+\tau_{1}\right), x\left(n+\tau_{2}\right), \cdots, x\left(n+\tau_{k-1}\right)\right\}
$$

The k-order moment $m_{k x}\left(\tau_{1}, \tau_{2}, \cdots, \tau_{k-1}\right)$ of this process is defined as the $\mathrm{k}$-order joint moment of random variable $\left\{x(n), x\left(n+\tau_{1}\right), \cdots, x\left(n+\tau_{k-1}\right)\right\}$, namely:

$$
m_{k x}\left(\tau_{1}, \tau_{2}, \cdots, \tau_{k-1}\right)=\operatorname{mom}\left\{x(n), x\left(n+\tau_{1}\right), \cdots, x\left(n+\tau_{k-1}\right)\right\}
$$

Here, mom() represents the joint moment, and the third-order cumulant is

$$
c_{3 x}\left(\tau_{1}, \tau_{2}\right)=E\left\{x(n) x\left(n+\tau_{1}\right) x\left(n+\tau_{2}\right)\right\}
$$

\section{Complex Third-Order Cumulant Slices}

According to literature (7), in Equation (3), $x(n)$ is the plural signal and is defined as follows:

Define one:

$$
c_{3 x}\left(\tau_{1}, \tau_{2}\right)=E\left\{x(n) x\left(n+\tau_{1}\right) x\left(n+\tau_{2}\right)\right\}
$$

Define two:

$$
c_{3 x}\left(\tau_{1}, \tau_{2}\right)=E\left\{x^{*}(n) x\left(n+\tau_{1}\right) x\left(n+\tau_{2}\right)\right\}
$$

Define three:

$$
c_{3 x}\left(\tau_{1}, \tau_{2}\right)=E\left\{x^{*}(n) x\left(n+\tau_{1}\right) x^{*}\left(n+\tau_{2}\right)\right\}
$$

where $x^{*}(n)$ is the conjugate complex number of $x(n)$, the complex signal in this paper is obtained by Hilbert transformation of the original signal collected. Literature (6), points out that the way to define a, can use the plural form of a harmonic of the quadratic phase coupling signals (such as type (7), the $\phi_{3}=\phi_{2}+\phi_{1}$, harmonic component $\omega_{3}$ is by the harmonic component of $\omega_{1}$ and $\omega_{2}$ by quadratic phase coupling, and $\left.\omega_{3}=\omega_{1}+\omega_{2}\right)$ and third order cumulant to 0 , the way to define the second and third order cumulant, as shown in the type (8) to define the three ways of third order cumulant as shown in type (9). 


$$
\begin{gathered}
x(n)=\sum_{i=1}^{3} A_{i} \exp \left(\omega_{i} n\right)+\phi_{i} \\
A_{1} A_{2} A_{3}\left[\exp \left[j\left(\omega_{1} \tau_{1}+\omega_{2} \tau_{2}\right)\right]+\exp \left[j\left(\omega_{2} \tau_{1}-\omega_{1} \tau_{2}\right)\right]\right] \\
A_{1} A_{2} A_{3}\left[\exp \left[j\left(\omega_{3} \tau_{1}-\omega_{1} \tau_{2}\right)\right]+\exp \left[j\left(\omega_{3} \tau_{1}-\omega_{2} \tau_{2}\right)\right]\right]
\end{gathered}
$$

Let $\tau_{2}=0$ in Equations ((8) and (9)) respectively, then Equation (8) becomes:

$$
A_{1} A_{2} A_{3}\left[\exp \left[j\left(\omega_{1} \tau_{1}\right)\right]+\exp \left[j\left(\omega_{2} \tau_{1}\right)\right]\right]
$$

then Equation (9) becomes:

$$
A_{1} A_{2} A_{3}\left[\exp \left[j\left(\omega_{3} \tau_{1}\right)\right]+\exp \left[j\left(\omega_{3} \tau_{1}\right)\right]\right]
$$

Can be seen from the type (3), when $\tau_{2}=0$ for the section, is the third order cumulants slices. Formulas (10) and (11) show that when the signal can be expressed in the form of complex harmonics, the third-order cumulant slices obtained by definition 2 and definition 3 contain only the previous-coupled signals and the after-coupled signals, respectively.

\section{Data Collection}

The pressure-reducing valve studied in this experiment is the pilot pressurereducing valve. When a foreign body oil press reducing valve in and out of the mouth, the different pressure will affect the normal operation of the valve's pressure, in order to get the pressure in operation under the fault state of the signal, this paper tests of artificial set the fault as follows:

Fault 1: add $\Phi 3 \mathrm{~mm}$ core to the relief valve outlet

Fault 2: $\Phi 3 \mathrm{~mm}$ iron core was added at the inlet and outlet of the pressure relief valve.

The working failure condition of pressure relief valve can be approximately simulated by experiments.

In this paper, LabVIEW software and pci-6014 data acquisition card and an acceleration sensor are used to collect vibration signals of pressure relief valve in normal and fault state successively. In each measurement, the oil pressure is divided into five pressure levels from $1 \mathrm{MPa}$ to $5 \mathrm{MPa}$. The sampling frequency is $250 \mathrm{~Hz}$, the reading frequency is $125 \mathrm{~Hz}$, and the sampling process took about 2 minutes. In this experiment, a total of 54 data sets of 18 groups were collected in the normal working state and two failure states of the pressure relief valve respectively. The number of data used in the experiment is 1536 .

Due to the system in the process of testing the influence of the external and internal factors inevitable in the process of the output with unwanted noise, In this paper, the median method is used to pre-process the collected vibration signal and eliminate the interference noise mixed in the signal. The filtered signal is shown in Figure 1. 


\section{Third Order Cross Section Calculation}

Fault recognition, this paper in order to will be measured in the experiments of normal state and failure state of the 36 sets of data, respectively, according to the type (4)-(6), and $\tau_{2}=0$, calculate the amount of plural third-order cumulant slices and take its modulus value, by contrast, let $-8 \leq \tau_{1} \leq 8$ and the calculated results are input support vector machine (SVM) to diagnose faults, A more satisfactory result can be obtained. Among them, the complex third-order cumulant slices calculated by definition 1 are shown in Table 1 .

\section{Experimental Result}

The same LSSVM is established in this paper. When trained LSSVM, respectively the normal state and fault state code is 1 and 1 , due to the normal data and fault data are obtained under the five different oil pressure, training respectively in table a normal data and fault data of different hydraulic conditions corresponding 10 groups, 5 sets of data to set up by the least squares support vector

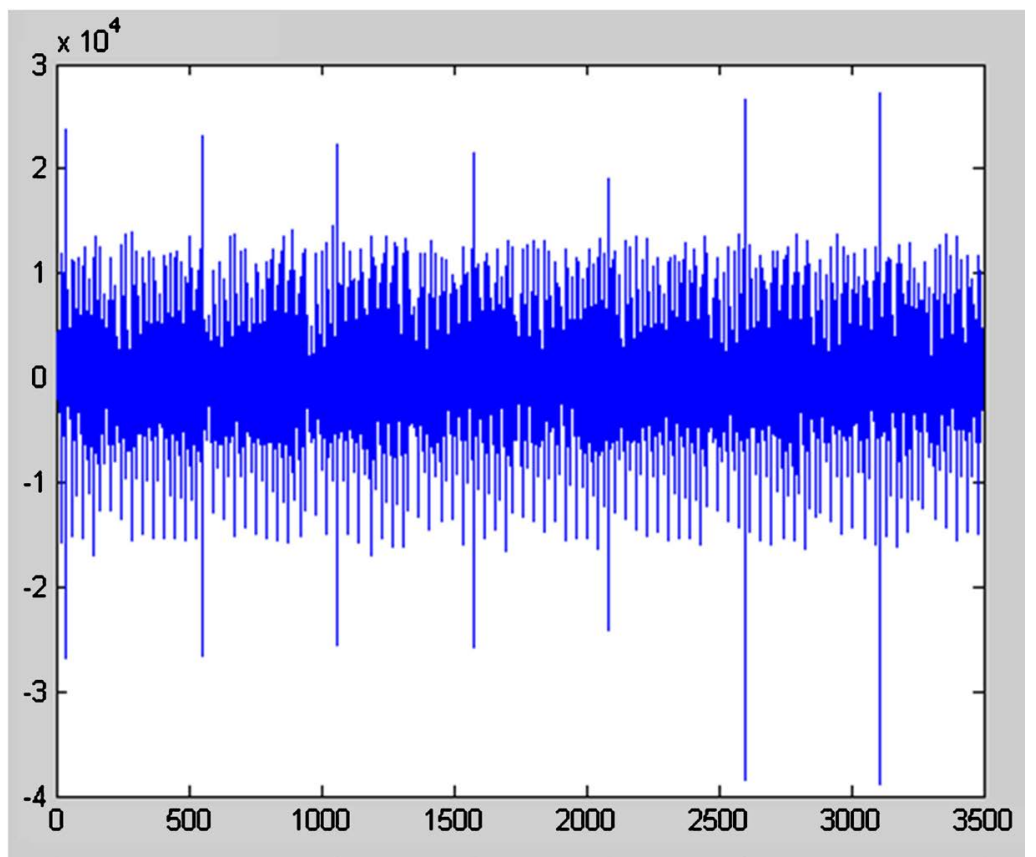

Figure 1. Filtered signal.

Table 1. The slice values of the third order of the normal state and failure state.

\begin{tabular}{cccc}
\hline & $1891,222.1180$ & $2,055,712.5807$ & $1,950,438.9033$ \\
$\begin{array}{c}\text { Normal } \\
\text { state }\end{array}$ & $724,726.0347$ & $549,599.1331$ & $525,556.0427$ \\
& $448,594.3487$ & $537,601.5387$ & $579,072.0499$ \\
\hline \multirow{2}{*}{$\begin{array}{c}\text { Failure } \\
\text { state }\end{array}$} & $3,935,434.0292$ & $2,980,882.4356$ & $3,161,106.0132$ \\
& $7,032,668.3428$ & $6,825,709.3659$ & $5,024,387.5584$ \\
\hline
\end{tabular}




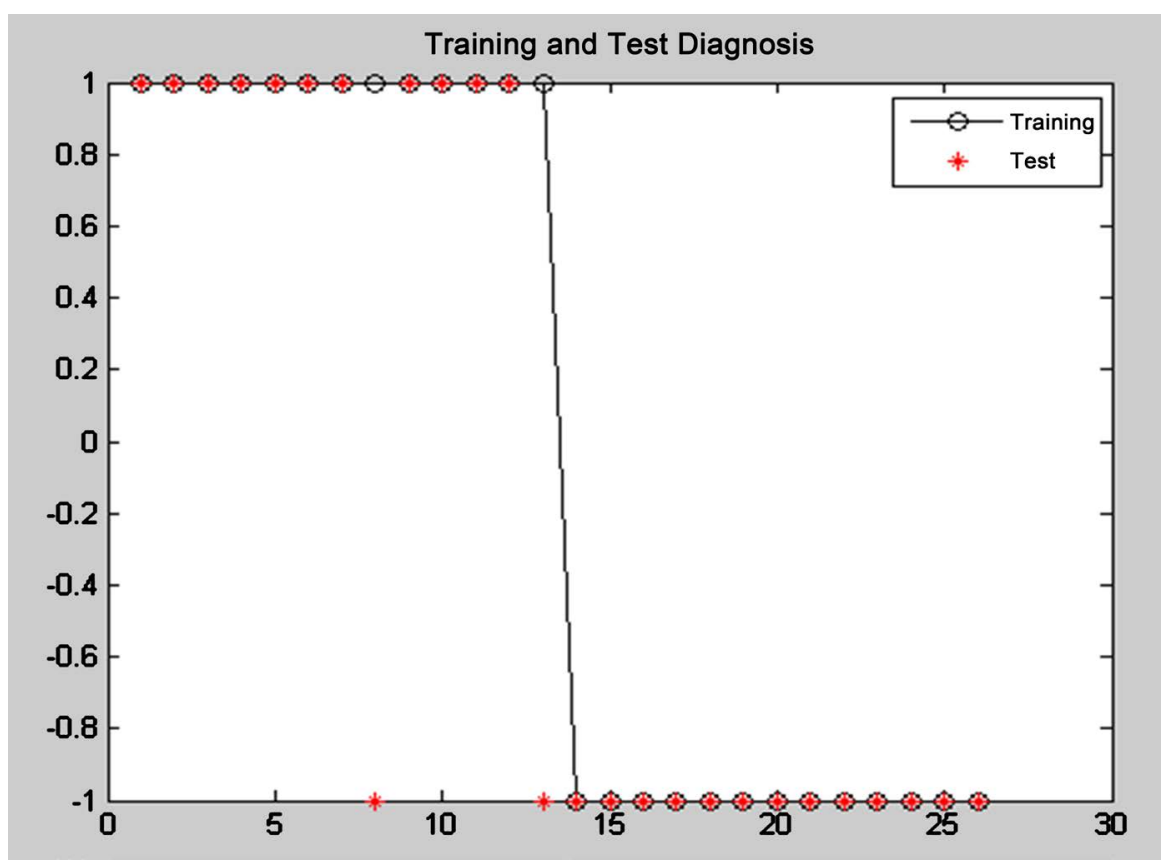

Figure 2. Results of the simulation of normal state and failure state in a defined way.

machine (SVM) training, training after recycling the support vector machine (SVM) is used to identify the fault, use the network after training for simulating the remnant of 26 sets of data to identify, as shown in Figure 2, which shows 26 sets of data after the simulation condition. The hollow circle represents the state of the training data set, such as the normal 13 sets of data, which should be in the state of 1 represented by a horizontal line above in the figure, the behind 13 groups of fault data should be in the state of -1 represented by another horizontal line below in the figure. If one hollow circle and one red solid circular belong to the same data set overlap, indicates the simulation results of this set of data is correct, otherwise error. The simulated results are shown in Figure 2. The results show that there are only 2 errors in the way of definition 1 , and the accuracy is over $90 \%$. In order to facilitate comparison, the same experiment was carried out according to the methods of definition 2 and definition 3 . The number of recognition errors was 2 groups and 4 groups respectively. At the same time, the data of fault 1 was changed to fault 2 , and the same experiment was conducted in three different definitions. The number of recognition errors was 3, 2 and 5 respectively.

\section{Spectrum}

In order to have an intuitive understanding of the properties of third-order cumulant slices in fault diagnosis based on the above 3 definitions, this paper defines three-order cumulant slice spectra as follows:

$$
\operatorname{Diag}(\omega)=\sum_{\tau_{1}=-\infty}^{\infty} c_{3 x}\left(\tau_{1}, 0\right) \mathrm{e}^{-j \omega \tau_{1}}
$$


In this experiment, 108 sets of third-order cumulant slices obtained from 36 sets of data in normal state and fault 1 state according to the 3 definitions mentioned above were calculated, and then the complex third-order cumulant slice spectrum according to formula (12) were calculated. In this paper, when the oil pressure is $1 \mathrm{MPa}, 3 \mathrm{MPa}$ and $5 \mathrm{MPa}$ respectively in normal state and failure state, a set of third-order cumulant slice spectra of the two states is selected and shown in Figures 3-8.
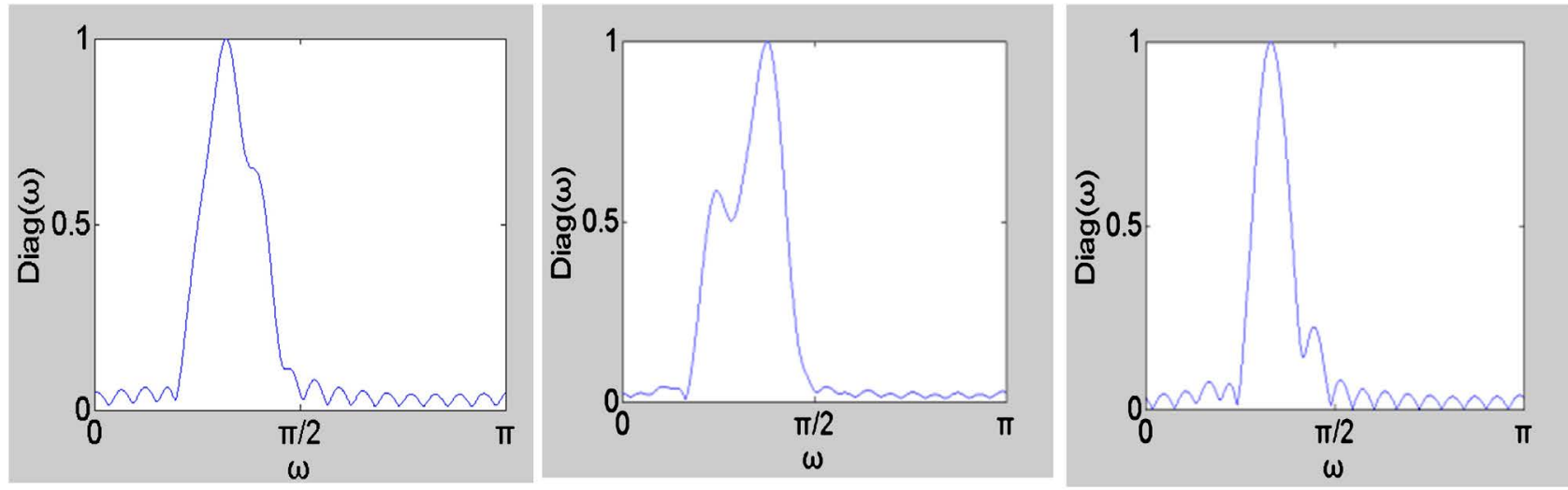

Figure 3. The normal state of a method is defined as the third order cross section spectrum.
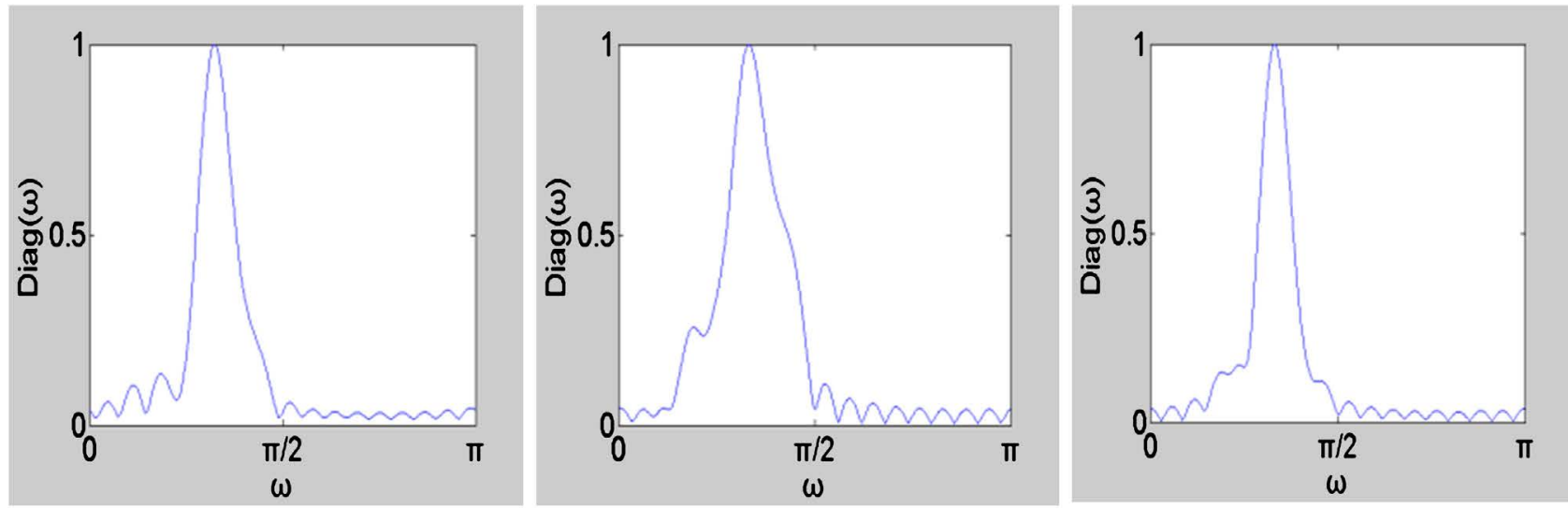

Figure 4. Defines a normal state of the third order cross section spectrum.
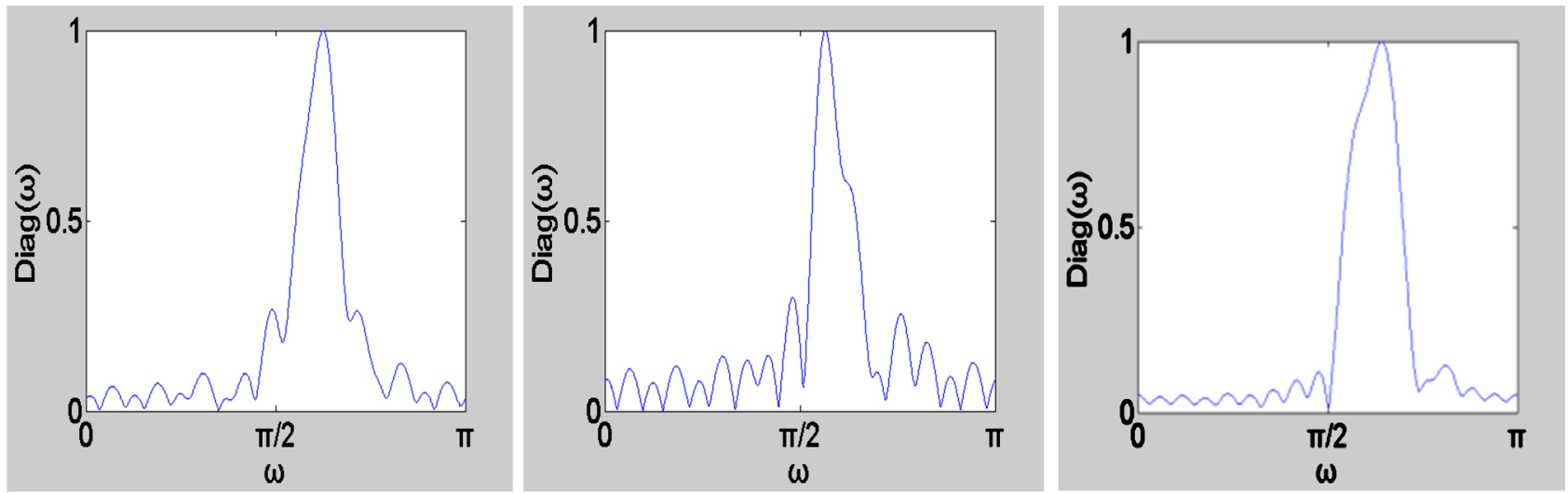

Figure 5. Define the normal state of the second mode and the third-order cross section spectrum. 

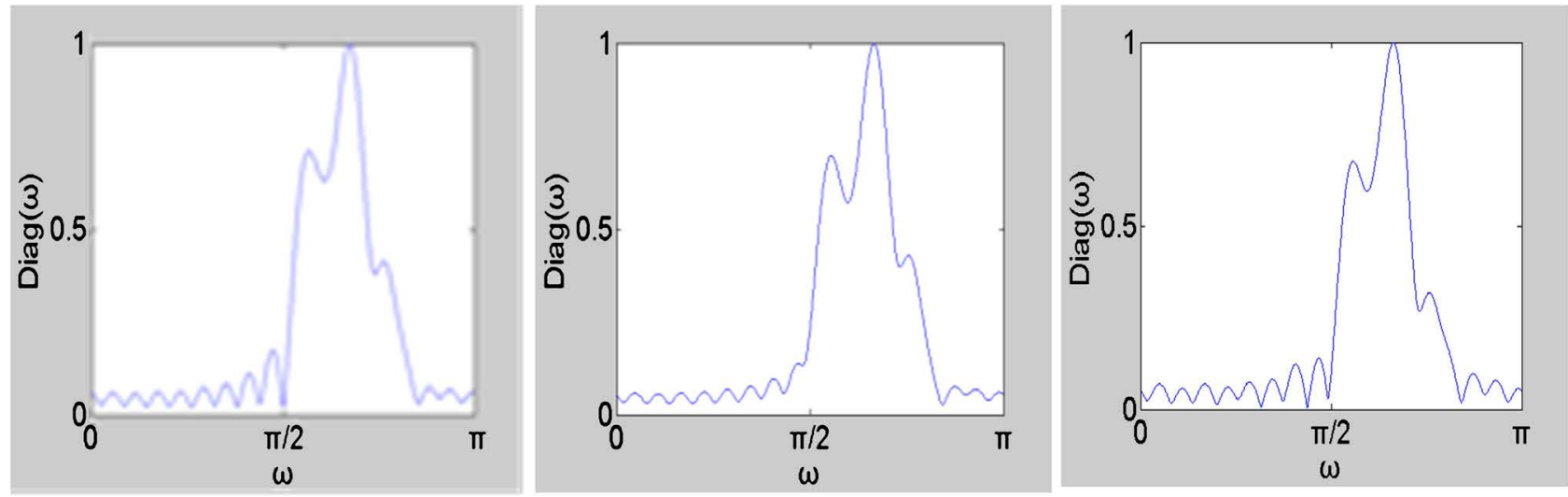

Figure 6. Define the fault state of the second mode and the third order cross section spectrum.
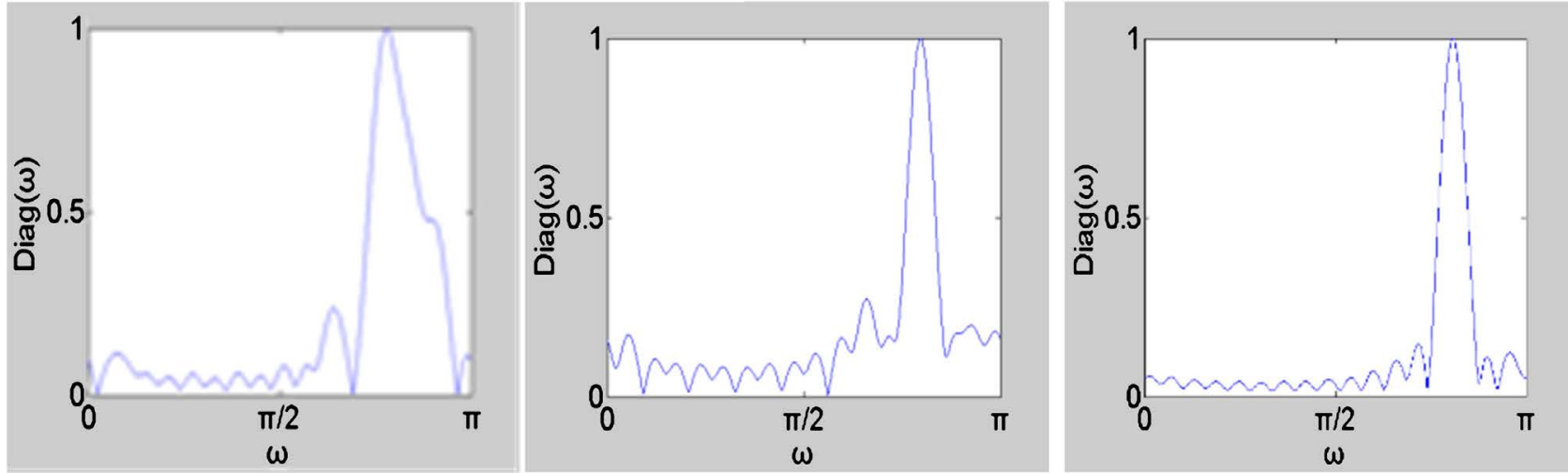

Figure 7. Define the normal state of the three-way normal third-order cross section spectrum.
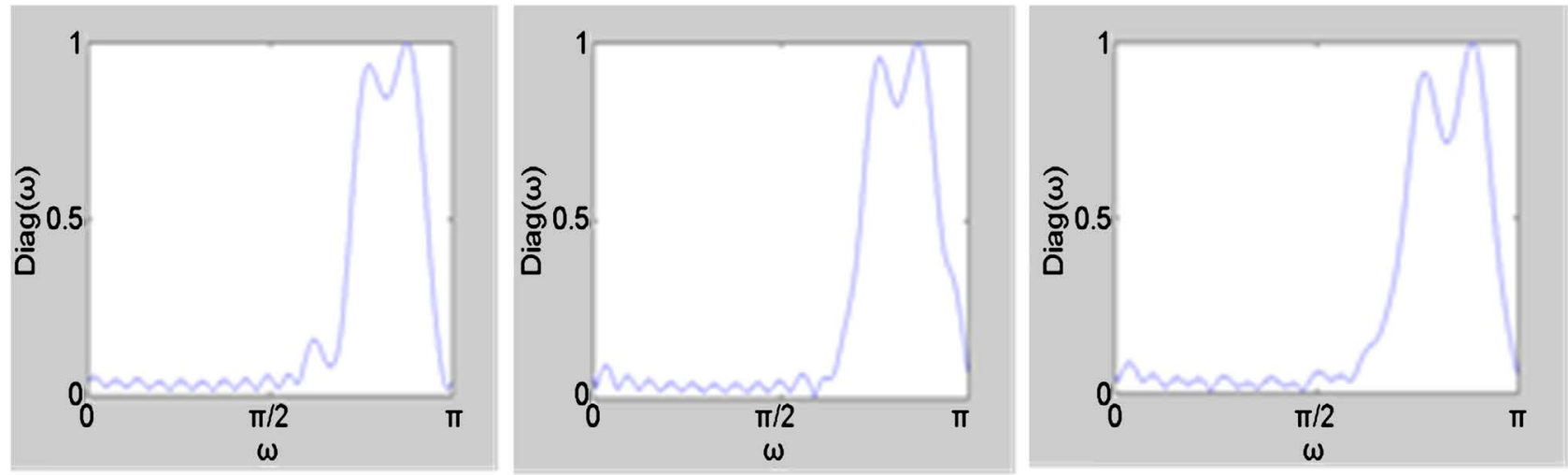

Figure 8. The third order cross section spectrum of fault state of three-way is defined.

In the figure, the $\mathrm{X}$-axis and $\mathrm{Y}$-axis represent the frequency in $\mathrm{HZ}$, and the vertical axis represents the normalized amplitude, without dimension. From Figure 3 to Figure 8, in general, defined in 3 ways, the amount of fault state of third-order cumulant slice spectrum compared with the normal condition, the distribution of the spectral peak become more complicated. At the same time, the distribution of the spectrum peaks of the third-order cumulant slices under definition 2 and definition 3 are more complicated than that under definition 1 , which should be due to the different coupling information contained in the 
different definitions mentioned above.

\section{Conclusion and Prospect}

Different definitions of complex third-order cumulants determine that they contain different coupling information, which is bound to be reflected in the complex third-order cumulant slices. In this paper, the different coupling characteristics are analyzed by fault diagnosis experiments. It is concluded that the ability of complex third-order cumulant slices with different coupling properties to maintain the characteristics of the same kind signals is different. It also provides an alternative method for fault diagnosis. The fault diagnosis method provided in this paper can be realized through the single-chip computer system, in which a certain amount of data can be stored first, and then the fault diagnosis can be made according to the data collected in real time.

\section{Acknowledgements}

This paper is supported by Research Foundation of the Nanchang Normal University for Doctors (NSBSJJ2018014), National Natural Science Foundation of China (61562063), Science and Technology Project of Jiangxi Provincial Education Department (GJJ171113).

\section{Conflicts of Interest}

The authors declare no conflicts of interest regarding the publication of this paper.

\section{References}

[1] Kocur, D. and Stanko, R. (2000) Order Bispectrum: A New Tool for Reciprocated Machine Condition Monitoring. Mechanical Systems and Signal Processing, 14, 871-890. https://doi.org/10.1006/mssp.2000.1307

[2] Cao, J.M., Shao, R.P. and Hu, W.T. (2011) Research on Damage Detection of Gear Combining HOC and EMD. Journal of Instrumentation, 32, 729-735.

[3] Shao, R.P. and Huang, X.N. (2008) Fault Detection and Diagnosis of Gear System Based on High Order Cumulant. Journal of Mechanical Engineering, 44, 161-168.

[4] Cai, Q.Z. and Huang, Y.J. (2009) Study on Autoregressive Trispectral Section. Journal of Instrumentation, 30, No. 2.

[5] Hinich, M.J. (1994) Higher-Order Cumulants and Cumulants Spectra. Circuits Systems and Signal Processing, 13, 391-420. https://doi.org/10.1007/BF01183737

[6] Raughveer, M.R. (1990) IEEE Trans. Time-Domain Approaches to Quadratic Phase Coupling Estimation. Automatic Control, 35, 48-56.

[7] Zhang, Y. and Wang, S.X. (1998) Nonlinear Phase-Coupled Method for SliceSpectrum Analysis. Electronic Journal, 26, 104-109.

[8] Zhang, Y., Wang, S.X. and Li, S.H. (1996) Dimensional $1 \frac{1}{2}$ Dimension Spectrum 1 Analysis of Secondary Phase Coupling. Electronic Journal, 64, 109-112. 